\title{
Desenvolvimento inicial do algodoeiro em resposta ao armazenamento de sementes tratadas com Cloreto de Mepiquat $^{1}$
}

\author{
Initial development of cotton in response to storage of seeds treated with Mepiquat \\ Chloride
}

\author{
Eliege Aparecida de Paiva Oliveira ${ }^{2 *}$, Claudemir Zucareli ${ }^{2}$, Celso Jamil Marur ${ }^{3}$, Getúlio Takashi Nagashima e $^{3}$ \\ Alberto Sérgio do Rego Barros ${ }^{3}$
}

\begin{abstract}
Resumo - O trabalho objetivou avaliar a manutenção do efeito do Cloreto de Mepiquat no desenvolvimento inicial das plantas de algodoeiro em função de doses e formas de aplicação do regulador e armazenamento das sementes tratadas. Sementes do cultivar IPR 120 foram tratadas com soluções de Cloreto de Mepiquat ( 250 g i.a. $\left.\mathrm{L}^{-1}\right)$ nas doses 0,$0 ; 5,0$; 10,$0 ; 15,0$ e 20,0 g i.a. $\mathrm{kg}^{-1}$ de sementes, via embebição por 12 horas e, via aplicação direta nas sementes. Ao zero; 60; 120 e 180 dias após a aplicação dos tratamentos as sementes foram semeadas em vasos e, semanalmente foram determinadas a altura e a área foliar e, aos 28 dias, o diâmetro do caule e matéria seca de caules e folhas. Os dados foram submetidos à análise de variância e a comparação de médias pelo teste de Tukey a 5\%, sob o delineamento inteiramente casualizado, com quatro repetições, em esquema fatorial de 5 x 2 (doses x formas de aplicação), independentemente dos períodos de armazenamento. As médias de doses foram submetidas a estudos de regressão. Independente da forma de aplicação, o Cloreto de Mepiquat reduz diâmetro do caule, matéria seca de caule e folhas, área foliar e altura de plantas. A forma de aplicação e o armazenamento das sementes tratadas por até 180 dias não interferem na ação do regulador. A ação reguladora de crescimento do Cloreto de Mepiquat perdura, pelo menos, até os 28 DAE das plantas, independente da forma de aplicação, dose utilizada e período de armazenamento das sementes tratadas.
\end{abstract}

Palavras - chave - Gossypium hirsutum L. Regulador vegetal. Embebição. Aplicação direta.

\begin{abstract}
The study evaluated the maintenance effect of Mepiquat chloride on the early development of cotton plants as a function of doses and application methods of regulating and storing seeds. The seeds were treated with 120 IPR solutions Mepiquat Chloride (250 g a.i. $\left.\mathrm{L}^{-1}\right)$ at doses of $0.0 ; 5.0 ; 10.0 ; 15.0$ and $20.0 \mathrm{~g}$ a.i. $\mathrm{kg}^{-1} \mathrm{seed}$, via soaking for 12 hours, and via direct application in seeds. At 0,60,120 and 180 days after the treatment the seeds were sown in pots and weekly were determined height and leaf area and, after 28 days, the stem diameter and dry mass of stems and leaves. Data were subjected to analysis of variance and comparisons of means by Tukey test at $5 \%$, under the completely randomized design with four replications in a factorial $5 \times 2$ (doses and application forms), independently, for each storage period.. The mean doses were subjected to regression studies. Independent of the application, Mepiquat Chloride reduces stem diameter, dry mass of stems and leaves, leaf area and plant height. The form of application and storage of seeds treated for 180 days did not interfere with the action of the regulator. The regulatory action of growth Mepiquat Chloride lasts at least until 28 DAE, independent of the application form, dose and storage period of seeds.
\end{abstract}

Key words - Gossypium hirsutum L. Plant growth regulator. Imbibition. Direct application.

\footnotetext{
* Autor para correspondência

${ }^{1}$ Recebido para publicação em 15/06/2010; aprovado em 25/04/2011

Parte da Dissertação do primeiro autor, apresentada ao Departamento de Ciências Agronômicas da Universidade Estadual de Londrina (UEL), financiada pela CAPES

${ }^{2}$ Universidade Estadual de Londrina, Brasil, paivaeliege@gmail.com, claudemircca@uel.br

${ }^{3}$ Instituto Agronômico do Paraná, Brasil, cjmarur@iapar.br, gtnagashima@iapar.br, asbarros@iapar.br
} 


\section{Introdução}

$\mathrm{O}$ algodoeiro tem crescimento indeterminado, com necessidade, em algumas situações de cultivo, limitar o crescimento dos órgãos vegetativos, para que ocorra partição de metabólitos favorável aos drenos úteis do ponto de vista econômico; botões florais, flores e maçãs (BELTRÃO et al., 1997).

O uso de regulador de crescimento é uma técnica eficaz, pois promove alterações na arquitetura das plantas, tornando-as mais compactas, facilitando a colheita mecanizada, permite o aumento da população de plantas por área, maior eficiência da pulverização de produtos químicos e favorece a penetração da luz, contribuindo para uma abertura mais rápida e uniforme dos frutos (ZANQUETA et al., 2004).

A redução no porte do algodoeiro está diretamente relacionada à dose aplicada do regulador de crescimento, sendo útil às culturas com perdas precoces de estruturas reprodutivas (shedding), causadas por ataques de pragas ou por algum fator de estresse que reduzem os drenos reprodutivos, de modo que os carboidratos são utilizados para o crescimento vegetativo (HOLDEN et al., 2004).

Entre as técnicas utilizadas na obtenção de plantas de algodão com menor porte, a aplicação de regulador de crescimento via tratamento de sementes, em soluções contendo Cloreto de Mepiquat, tem sido recentemente pesquisada e, com resultados promissores. A vantagem em relação à aplicação foliar é que a planta terá seu crescimento controlado desde a emergência, sem riscos de perdas do produto por lavagem após ocorrência de chuvas e por deriva em pulverizações foliares (MATEUS et al., 2004; NAGASHIMA et al., 2005).

Yeates et al. (2005), compararam tratamentos de sementes de algodão com regulador de crescimento via embebição e aplicação direta na semente, em condições de campo, e observaram que ambas as formas de aplicação do regulador reduziram precocemente o porte das plantas. Segundo os autores a redução e a duração do efeito redutor estão diretamente relacionadas à concentração utilizada.

Nagashima et al. (2010), ao conduzirem experimento em casa de vegetação utilizando sementes de algodão da linhagem PR 02-307 sem tratamento e com aplicação direta de Cloreto de Mepiquat nas concentrações de 3,75 e 7,5 g i.a. $\mathrm{kg}^{-1}$ sobre as sementes e embebidas em solução com as mesmas concentrações, observaram que o Cloreto de Mepiquat pode ser usado no tratamento de sementes, tanto por embebição quanto por aplicação direta e, independente da concentração, os efeitos são semelhantes na redução da altura e crescimento dos ramos do algodoeiro, com efeito mais acentuado com o aumento das concentrações dentro da faixa estudada.
Por ser uma técnica relativamente recente, são escassas as informações relacionadas ao tratamento de sementes de algodão com reguladores de crescimento, em função de doses, formas de aplicação e, principalmente, no que se refere à manutenção do efeito regulador do produto após o armazenamento das sementes já tratadas.

Sementes armazenadas após determinado período, além da perda da compartimentalização celular e da desintegração do sistema de membranas, promove descontrole do metabolismo e das trocas de água e solutos entre as células e o meio exterior, determinando a redução da viabilidade da semente (MARCOS FILHO, 1999). Essas são algumas evidências de que danos às membranas celulares resultam em menor habilidade de reter os solutos dentro das células, podendo ser um dos efeitos do armazenamento prolongado.

Neste contexto, objetivou-se com esse trabalho avaliar a manutenção do efeito do Cloreto de Mepiquat no desenvolvimento inicial das plantas de algodoeiro em função de doses e formas de aplicação deste regulador e armazenamento das sementes tratadas.

\section{Material e métodos}

O experimento foi conduzido em casa de vegetação, nas instalações do Departamento de Agronomia, da Universidade Estadual de Londrina (UEL), LondrinaPR, (2329'41,4” S e $51^{\circ} 12^{\prime} 5,5$ ” W), durante o período de dezembro de 2008 a julho de 2009. O delineamento experimental utilizado foi inteiramente casualizado, em esquema fatorial $5 \times 2$, correspondente a cinco doses de Cloreto de Mepiquat e duas formas de aplicação (embebição e aplicação direta), totalizando 10 tratamentos, com quatro repetições.

Foram utilizadas sementes de algodão deslintadas quimicamente com ácido sulfúrico do cultivar IPR 120. As sementes foram embebidas em solução de Cloreto de Mepiquat (250 g i.a $\mathrm{L}^{-1}$ ) nas doses de 0,$0 ; 5,0 ; 10,0$; 15,0 e 20,0 g i.a kg-1 de sementes, por um período de 12 horas a $20{ }^{\circ} \mathrm{C}$, sendo utilizados $400 \mathrm{~mL}$ de solução por $\mathrm{kg}$ de sementes. Para o tratamento via aplicação direta, foram utilizadas as mesmas doses do regulador de crescimento, contudo, com aplicação externa a semente. As sementes foram acondicionadas em saco plástico para agitação até distribuição uniforme, utilizando $100 \mathrm{~mL}$ de solução por kg de sementes.

Após a aplicação do regulador, as sementes foram secas a sombra em local ventilado e, acondicionadas em sacos de papel tipo "Kraft" e armazenadas por até 180 dias, em condições ambientais não controladas. Os dados de temperatura e umidade relativa (máxima 
e mínima) do ar durante o período de armazenamento, obtidos com aparelho termohigrógrafo modelo R-704, estão apresentados na Figura 1.

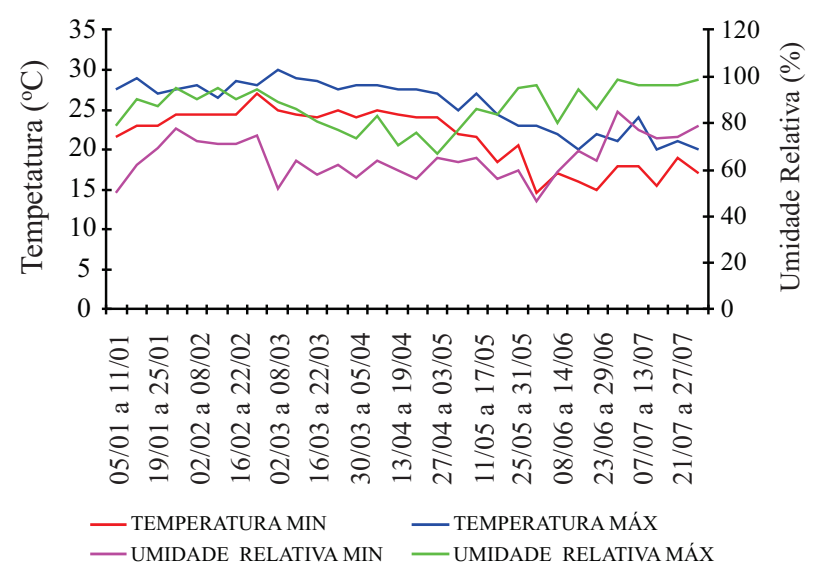

Figura 1 - Dados de temperatura $\left({ }^{\circ} \mathrm{C}\right)$ e umidade relativa do ar (\%) durante o período do armazenamento das sementes de algodão sob condições ambientais não controladas

Para verificar a manutenção do efeito do regulador de crescimento, foi avaliado o desenvolvimento das plantas em casa de vegetação, logo após o tratamento e a cada 60 dias após o início do armazenamento. Para tanto, foram semeadas cinco sementes de algodão cultivar IPR 120, em vasos com capacidade de 2,5 L, preenchidos com mistura contendo $40 \%$ de solo, $30 \%$ de palha de arroz carbonizada e $30 \%$ de areia e adubado com 5,0 g de NPK 8-28-16 em cada vaso. O desbaste foi realizado sete dias após a emergência das plântulas, deixando apenas uma planta por vaso.

A cada sete dias após a emergência (DAE) das plântulas, até os 28, foram avaliadas a altura e a área foliar das plantas. Aos $28 \mathrm{DAE}$, no final do experimento, foram ainda determinados: o diâmetro do caule, a altura de inserção do nó cotiledonar e a massa de matéria seca de caules e folhas, conforme metodologias descritas a seguir: Altura de Planta: Para esta determinação foi tomado o comprimento das plantas, em centímetros, da distância entre o nível do solo ao ápice da planta, com o auxílio de régua graduada. Área Foliar Estimada: foi utilizada a equação $\mathrm{Y}=0,8529 \mathrm{X}\left(\mathrm{R}^{2}=0,98\right)$, em que $\mathrm{Y}$ é a área estimada e $\mathrm{X}$ o produto do comprimento e largura de cada folha. Para as folhas cotiledonares e primeiras folhas verdadeiras cordiformes, foram consideradas as larguras e comprimentos máximos; nas folhas subsequentes, lobadas, a largura foi obtida na posição final da nervura dos lobos basais, e o comprimento avaliado desde a inserção da folha no pecíolo até o ápice da folha, de acordo com a metodologia descrita por Nagashima et al. (2005). Diâmetro do Caule: Determinado com o auxílio de paquímetro, a um centímetro da superfície do solo. Matéria Seca: Para determinação da matéria seca $(\mathrm{mg})$ de folhas e caules, os materiais foram acondicionados em sacos de papel e levados à estufa de circulação de ar forçada $65 \pm 5^{\circ} \mathrm{C}$ até a obtenção da massa constante. Em seguida, foram pesadas em balança com precisão de $0,01 \mathrm{~g}$.

Os dados isoladamente para cada período de armazenamento foram submetidos à análise de variância pelo teste $\mathrm{F}$ a $5 \%$ de significância. As médias de formas de aplicação foram comparadas pelo teste de Tukey a 5\% e as médias de doses submetidas a estudos de regressão até $2^{\circ}$ grau.

\section{Resultados e discussão}

O efeito de forma de aplicação foi constatado nas avaliações de altura de plantas e área foliar ao zero e 180 dias após o armazenamento (DAA). Nas avaliações de altura de plantas e área foliar realizadas aos 7; 14; $21 \mathrm{e}$ 28 dias após a emergência (DAE), a análise de variância demonstrou o efeito significativo para doses do regulador utilizadas no tratamento de sementes, independente da forma de aplicação, mantendo o efeito redutor significativo até o final do período de armazenamento. Ocorreu interação para altura de plantas nos períodos de zero, 60 e 120 DAA e para área foliar nos períodos de zero e 120 DAA, não sendo constatada interação entre os outros fatores analisados (TAB. 1).

As formas de aplicação de Cloreto de Mepiquat avaliadas não diferiram com relação à redução na altura de plantas nos períodos de zero, 60 e 120 DAA (TAB. 2), sendo os efeitos concordantes com os resultados obtidos por Nagashima et al. (2010), os quais observaram que o Cloreto de Mepiquat pode ser usado no tratamento de sementes, tanto por embebição quanto por aplicação direta e, independente da concentração utilizada $(3,75$ ou $7,5 \mathrm{~g}$ de i.a $\mathrm{kg}^{-1}$ ), os efeitos são semelhantes na redução da altura do algodoeiro, com efeito, mais acentuado com o aumento das doses utilizadas. Aos 180 DAA observou-se diferença significativa entre as formas de aplicação nas avaliações realizadas aos 7 e 14 DAE das plantas, com menores valores de altura no tratamento via aplicação direta (A.D.) em relação à embebição (EMB.). Esses resultados diferem do obtido por Yeates et al. (2005), que ao conduzirem dois experimentos para avaliar em condições de campo o efeito do tratamento de sementes com Cloreto de Mepiquat, via embebição, por 2,5 horas, e aplicação direta do produto, 
Tabela 1 - Resumo da análise de variância $(\mathrm{F}<0,05)$ para os fatores formas de aplicação, doses, interação forma e dose para altura de plantas (AP), área foliar (AF) avaliadas aos 7; 14; 21 e 28 dias após emergência (DAE) e diâmetro do caule (DC), matéria seca de caule (MSC) e matéria seca de folhas (MSF) aos 28 dias em plantas de algodoeiro, do cultivar IPR 120, originadas de sementes tratadas com Cloreto de Mepiquat em função de formas e doses em diferentes períodos de armazenamento

\begin{tabular}{|c|c|c|c|c|c|c|c|c|c|c|c|}
\hline \multicolumn{12}{|c|}{ ZERO DIA APÓS O ARMAZENAMENTO } \\
\hline FA & AP 07d & AP 14d & AP 21d & AP 28d & AF 07d & AF $14 d$ & AF 21d & AF 28d & $\mathrm{DC} / \mathrm{mm}$ & MSC & MSF \\
\hline Forma & $0,671^{\text {ns }}$ & $0,976^{\text {ns }}$ & $0,893^{\text {ns }}$ & $0,819^{\text {ns }}$ & $0,040 *$ & $0,038^{*}$ & $0,059^{\text {ns }}$ & $0,004 * *$ & $0,460^{\text {ns }}$ & $0,454^{\mathrm{ns}}$ & $0,205^{\mathrm{ns}}$ \\
\hline Doses & $0,000^{* *}$ & $0,000 * *$ & $0,000 * *$ & $0,001 * *$ & $0,038^{*}$ & $0,000 * *$ & $0,000 * *$ & $0,012 *$ & $0,566^{\mathrm{ns}}$ & $0,010^{*}$ & $0,008 *$ \\
\hline orma*Dose & $0,094^{\mathrm{ns}}$ & $0,134^{\mathrm{ns}}$ & $0,100^{\mathrm{ns}}$ & $0,079^{\text {ns }}$ & $0,002 * *$ & $0,006^{*}$ & $0,005^{* *}$ & $0,000^{* *}$ & $0,115^{\text {ns }}$ & $0,148^{\mathrm{ns}}$ & $0,044^{\mathrm{ns}}$ \\
\hline $\mathrm{CV} \%$ & 19,01 & 25,99 & 27,12 & 29,08 & 27,39 & 33,78 & 40,73 & 43,17 & 17,39 & 48,19 & 37,68 \\
\hline \multicolumn{12}{|c|}{60 DIAS APÓS O ARMAZENAMENTO } \\
\hline Forma & 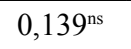 & $0,144^{\mathrm{ns}}$ & $0,512^{\mathrm{ns}}$ & $0,116^{\text {ns }}$ & $0,730^{\mathrm{ns}}$ & $0,616^{\mathrm{ns}}$ & $0,322^{\mathrm{ns}}$ & & & $0,052^{\text {ns }}$ & $0,234^{\mathrm{ns}}$ \\
\hline Doses & $0,000^{* *}$ & $0,000 * *$ & $0,000 * *$ & $0,000 * *$ & $0,014 *$ & $0,004 * *$ & $0,000 * *$ & $0,001 * *$ & $0,064^{\mathrm{ns}}$ & $0,172^{\text {ns }}$ & $0,127^{\mathrm{ns}}$ \\
\hline orma*Dose & $0,044 *$ & $0,275^{\mathrm{ns}}$ & $0,459^{\text {ns }}$ & $0,397^{\mathrm{ns}}$ & $0,481^{\mathrm{ns}}$ & $0,580^{\mathrm{ns}}$ & $0,113^{\mathrm{ns}}$ & $0,636^{\mathrm{ns}}$ & $0,357^{\mathrm{ns}}$ & $0,512^{\mathrm{ns}}$ & $0,404^{\mathrm{ns}}$ \\
\hline $\mathrm{CV} \%$ & 15,07 & 16,03 & 17,76 & 17,15 & 20,04 & 19,69 & 25,24 & 30,26 & 16,93 & 28,06 & 32,52 \\
\hline \multicolumn{12}{|c|}{120 DIAS APÓS O ARMAZANAMENTO } \\
\hline Forma & $0,897^{\mathrm{ns}}$ & $0,785^{\text {ns }}$ & $0,512^{\mathrm{ns}}$ & $0,722^{\mathrm{ns}}$ & $0,325^{\mathrm{ns}}$ & $0,656^{\mathrm{ns}}$ & $0,864^{\mathrm{ns}}$ & $0,375^{\text {ns }}$ & $0,034^{*}$ & $0,253^{\mathrm{ns}}$ & $0,620^{\mathrm{ns}}$ \\
\hline Doses & $0,018 *$ & $0,045^{*}$ & $0,002 * *$ & $0,017 *$ & $0,000 * *$ & $0,108^{\mathrm{ns}}$ & $0,019^{*}$ & $0,027^{*}$ & $0,000 * *$ & $0,437^{\mathrm{ns}}$ & $0,005^{* *}$ \\
\hline orma*Dose & $0,035^{*}$ & $0,052 *$ & $0,689^{\text {ns }}$ & $0,175^{\text {ns }}$ & $0,017 *$ & $0,832^{\mathrm{ns}}$ & $0,689^{\mathrm{ns}}$ & $0,747^{\mathrm{ns}}$ & $0,391^{\mathrm{ns}}$ & $0,455^{\text {ns }}$ & $0,581^{\mathrm{ns}}$ \\
\hline $\mathrm{CV} \%$ & 5,59 & 15,44 & 16,5 & 15,45 & 18,65 & 21,14 & 25,34 & 40,43 & 1,95 & 55,76 & 43,05 \\
\hline \multicolumn{12}{|c|}{180 DIAS APÓS O ARMAZENAMENTO } \\
\hline Forma & $0,032 *$ & $0,022 *$ & $0,273^{\text {ns }}$ & $0,210^{\text {ns }}$ & $0,288^{\text {ns }}$ & $0,116^{\mathrm{ns}}$ & $0,075^{\text {ns }}$ & $0,110^{\text {ns }}$ & $0,419^{\text {ns }}$ & $0,067^{\text {ns }}$ & $0,378^{\text {ns }}$ \\
\hline Doses & $0,005 * *$ & $0,000 * *$ & $0,000 * *$ & $0,000 * *$ & $0,544^{\mathrm{ns}}$ & $0,009 *$ & $0,000^{* *}$ & $0,000 * *$ & $0,689^{\text {ns }}$ & $0,000 * *$ & $0,020^{*}$ \\
\hline orma*Dose & $0,703^{\text {ns }}$ & $0,235^{\mathrm{ns}}$ & $0,225^{\mathrm{ns}}$ & $0,244^{\mathrm{ns}}$ & $0,787^{\text {ns }}$ & $0,895^{\mathrm{ns}}$ & $0,301^{\mathrm{ns}}$ & $0,286^{\mathrm{ns}}$ & $0,503^{\text {ns }}$ & $0,411^{\mathrm{ns}}$ & $0,762^{\mathrm{ns}}$ \\
\hline CV\% & 59,1 & 22,91 & 28,98 & 34,64 & 63,49 & 33,1 & 47,52 & 52,53 & 30,29 & 53,81 & 48,39 \\
\hline
\end{tabular}

* e **: significativo a $5 \%$ e $1 \%$, respectivamente. ns: não significativo

verificaram que o método da embebição causou o dobro da redução da altura quando comparado com o método da aplicação direta.

Para altura de planta em função das doses, independentemente da formas de tratamento utilizado, foram observadas redução de porte em todos os tratamentos com aplicação de Cloreto de Mepiquat em relação à testemunha (dose 0), desde a emergência até os 28 DAE, para todos os períodos de armazenamento. Quanto maior a concentração do regulador de crescimento aplicado, maior foi a redução na altura da planta (TAB. 2). Neste aspecto, os resultados obtidos neste estudo corroboram com os observados por Yeates et al. (2005), ao relatarem que o tratamento de sementes de algodão com regulador de crescimento, independentemente da forma, é útil na redução precoce do porte das plantas. Nagashima et al. (2009), ao conduzirem experimento em condições de campo, utilizando sementes de algodão embebidas por 12 horas em soluções contendo Cloreto de Mepiquat nas doses 0,0; 3,75; 7,5 e 15 g i.a. kg-1 de sementes, também constataram redução na altura de plantas de algodoeiro, com efeito visível até os 80 DAE, sendo o efeito relacionado com o aumento da dose utilizada.

A altura da planta (TAB. 2), aos 7 DAE, já demonstrou o efeito de doses do regulador, ajustando-se a uma equação linear decrescente com o incremento das doses ao zero e 180 DAA das sementes tratadas. Aos 14 DAE, o ajuste foi quadrático em função do aumento das doses para todos os períodos de armazenamento analisados, com pontos de mínima resposta de $18,50 \mathrm{~g}$ i.a. $\mathrm{kg}^{-1}$ ao zero DAA, de 15,39 aos 60 DAA e, aos 120 DAA 14,71 e de 15,42 aos 180 DAA.

Aos 21 DAE a altura de plantas ajustou-se a uma equação quadrática em função do aumento das doses para a maioria dos períodos analisados, com pontos de mínima em 15,27 g i.a. $\mathrm{kg}^{-1}$ aos 60 DAA, 12,00 aos 120 DAA e de 14,74 aos 180 DAA, com exceção do período de zero DAA no qual, o ajuste foi linear decrescente.

A altura de plantas aos 28 DAE, ao zero DAA, ajustou-se a uma equação linear decrescente. Nos demais 
Tabela 2 - Altura média (AP/cm) avaliada aos 7; 14; 21 e 28 dias após emergência (DAE) em plantas de algodoeiro, cultivar IPR 120, oriundas de sementes tratadas com Cloreto de Mepiquat em função de formas e doses em diferentes períodos de armazenamento

\begin{tabular}{|c|c|c|c|c|c|c|c|c|c|c|}
\hline \multicolumn{11}{|c|}{ ZERO DIA APÓS O ARMAZENAMENTO } \\
\hline \multirow{2}{*}{ Variáveis } & \multicolumn{2}{|c|}{ FORMAS } & \multicolumn{5}{|c|}{ DOSES (g i.a kg-1) } & \multirow[b]{2}{*}{ Equações de Regressão } & \multirow{2}{*}{$\mathrm{R}^{2}$} & \multirow{2}{*}{$\begin{array}{c}\text { P. } \\
\text { Mín. }\end{array}$} \\
\hline & EMB. & A.D. & 0 & 5 & 10 & 15 & 20 & & & \\
\hline AP07d & $5,74 \mathrm{a}$ & $5,89 \mathrm{a}$ & 7,74 & 6,15 & 5,25 & 5,73 & 4,24 & $\mathrm{Y}=7,305-0,149^{*} \mathrm{x}$ & 0,83 & - \\
\hline AP14d & $10,08 \mathrm{a}$ & $10,05 \mathrm{a}$ & 14,99 & 10,61 & 8,41 & 9,44 & 6,89 & $\mathrm{Y}=14,525-0,740 * \mathrm{x}-0,020^{*} \mathrm{x}^{2}$ & 0,89 & 18,50 \\
\hline AP21d & 14,48 a & $14,65 \mathrm{a}$ & 21,01 & 15,99 & 11,49 & 14,35 & 10,01 & $\mathrm{Y}=19,298-0,473 * \mathrm{x}$ & 0,76 & - \\
\hline AP28d & $18,83 \mathrm{a}$ & $19,23 \mathrm{a}$ & 25,88 & 21,65 & 14,63 & 19,44 & 13,58 & $Y=24,395-0,536^{*} x$ & 0,70 & - \\
\hline \multicolumn{11}{|c|}{60 DIAS APÓS O ARMAZENAMENTO } \\
\hline AP14d & $11,89 \mathrm{a}$ & $11,25 \mathrm{a}$ & 16,01 & 12,55 & 10,91 & 11,01 & 10,78 & $Y=15,823-0,708 * x+0,023 * x^{2}$ & 0,97 & 15,39 \\
\hline AP21d & $16,49 \mathrm{a}$ & $16,53 \mathrm{a}$ & 23,45 & 16,78 & 13,1 & 13,29 & 12,98 & $\mathrm{Y}=23,17-1,436^{*} \mathrm{x}+0,047^{*} \mathrm{x}^{2}$ & 0,98 & 15,27 \\
\hline AP28d & $19,97 \mathrm{a}$ & $19,23 \mathrm{a}$ & 26,1 & 24,14 & 19,49 & 18,94 & 18,61 & $Y=26,543-0,824 * x+0,021 * x^{2}$ & 0,95 & 19,61 \\
\hline \multicolumn{11}{|c|}{120 DIAS APÓS O ARMAZENAMENTO } \\
\hline AP14d & $9,84 \mathrm{a}$ & $9,89 \mathrm{a}$ & 13,39 & 12,93 & 12,23 & 11,88 & 12,59 & $Y=13,518-0,206^{*} x+0,007 * x^{2}$ & 0,88 & 14,71 \\
\hline AP21d & $11,26 \mathrm{a}$ & $10,98 \mathrm{a}$ & 15,1 & 13,76 & 13,34 & 13,88 & 14,01 & $Y=14,987-0,264 * x+0,011 * x^{2}$ & 0,88 & 12,00 \\
\hline AP28d & $12,26 \mathrm{a}$ & $12,33 \mathrm{a}$ & 17,76 & 15,85 & 14,06 & 14,79 & 14,68 & $\mathrm{Y}=17,746-0,494 * \mathrm{x}-0,017 * \mathrm{x}^{2}$ & 0,93 & 15,52 \\
\hline \multicolumn{11}{|c|}{180 DIAS APÓS O ARMAZENAMENTO } \\
\hline AP07d & $2,10 \mathrm{a}$ & $1,38 \mathrm{~b}$ & 2,89 & 2,08 & 1,69 & 0,91 & 1,14 & $Y=2,673-0,093 * x$ & 0,87 & - \\
\hline AP14d & $4,03 \mathrm{a}$ & $3,38 \mathrm{~b}$ & 7,03 & 3,61 & 3,10 & 2,43 & 2,39 & $Y=6,744-0,586^{*} x+0,019^{*} x^{2}$ & 0,95 & 15,42 \\
\hline AP21d & $5,19 \mathrm{a}$ & $4,68 \mathrm{a}$ & 10,44 & 4,69 & 3,45 & 3,06 & 3,05 & $\mathrm{Y}=9,978-1,032 * \mathrm{x}+0,035^{*} \mathrm{x}^{2}$ & 0,95 & 14,74 \\
\hline AP28d & $7,17 \mathrm{a}$ & $6,23 \mathrm{a}$ & 15,46 & 5,96 & 4,04 & 4,10 & 3,96 & $Y=14,636-1,681 * x+0,059 * x^{2}$ & 0,94 & 14,24 \\
\hline
\end{tabular}

Médias seguidas de mesma letra na linha, para efeito de formas de aplicação, não diferem pelo teste de Tukey a $5 \%$ de significância; * Significativo a 5\%; Embebição (EMB.); Aplicação Direta (A.D.); Ponto de Mínima (P. Mín.)

períodos de armazenamento ajustaram-se a uma equação quadrática com elevação das doses do regulador de crescimento com ponto de mínima de 19,61 g i.a. kg-1 aos 60 DAA, 15,52 aos 120 DAA e 14,24 g i.a. kg-1 aos 180 DAA. Resultados semelhantes a estes também foram constatados por Nagashima et al. (2005) no qual, em estudos avaliados até 49 DAE, verificaram que a altura da planta de algodoeiro diminui com o aumento da concentração do princípio ativo na solução aplicada. Lamas et al. (2006) ao utilizar a aspersão direta nas sementes, constataram que o efeito da ação do regulador foi mantido até o início do florescimento.

Para a variável altura de planta $(\mathrm{AP} / \mathrm{cm})$ aos 07 DAE com 60 dias de armazenamento das sementes tratadas (TAB. 3), foi constatada interação entre os fatores formas de aplicação e doses de Cloreto de Mepiquat.

Observou-se diferença significativa entre as formas de aplicação do regulador apenas na dose de 15 g i.a. $\mathrm{kg}^{-1}$, com menor altura de plantas para o tratamento via aplicação direta, quando comparado com o tratamento via embebição. Nas demais doses avaliadas não ocorreram diferenças entre as formas de aplicação. Com relação às doses, a resposta da planta em relação à altura ajustouse ao modelo quadrático para ambos os tratamentos, com ponto mínimo de $15,82 \mathrm{~g}$ i.a. $\mathrm{kg}^{-1}$ para o tratamento via embebição e, 15,95 g i.a. $\mathrm{kg}^{-1}$ para o tratamento via aplicação direta. Resultados estes que se assemelham aos obtidos por Nagashima et al. (2005), Nagashima et al. (2007) e Nagashima et al. (2009) que, em experimentos em casa de vegetação e campo utilizando sementes de algodão embebidas em soluções com Cloreto de Mepiquat, observaram plantas com menor estatura, com efeito, persistindo até 31 (2007) e 80 DAE (2009), em condições de campo, sendo a redução relacionada com o aumento da concentração utilizada. Yeates et al. (2005), Holden et al. (2004) e Lamas et al. (2000) também relatam que maior dose de regulador tende à redução mais acentuada no porte das plantas de algodoeiro, contudo, utilizando o Cloreto de Mepiquat via pulverização foliar.

Na Tabela 3 são apresentados os dados de altura de plantas avaliados aos 7 DAE, com 120 DAA das sementes tratadas, na qual foi observada, para a forma de aplicação, diferença significativa entre os tratamentos utilizados na dose de $20 \mathrm{~g}$ i.a $\mathrm{kg}^{-1}$, sendo 
Tabela 3 - Interação entre formas (FA) e doses para médias de altura (AP/cm) avaliadas aos 07 DAE em plantas de algodoeiro, cultivar IPR 120, oriundas de sementes tratadas com Cloreto de Mepiquat aos 60 e 120 dias de armazenamento

\begin{tabular}{|c|c|c|c|c|c|c|c|c|}
\hline \multicolumn{9}{|c|}{60 DIAS APÓS O ARMAZENAMENTO } \\
\hline AP7d & \multicolumn{5}{|c|}{ DOSES (g i.a. kg ${ }^{-1}$ ) } & & \multirow{2}{*}{$\mathrm{R}^{2}$} & \multirow{2}{*}{ P. Mín. } \\
\hline FA & 0 & 5 & 10 & 15 & 20 & Equações de Regressão & & \\
\hline Embebição & 8,70 a & $6,96 \mathrm{a}$ & $5,93 \mathrm{a}$ & 6,43 a & $5,94 \mathrm{a}$ & $Y=8,822-0,443 * x+0,014 * x^{2}$ & 0,99 & 15,82 \\
\hline Aplicação direta & $8,78 \mathrm{a}$ & $7,03 \mathrm{a}$ & $5,98 \mathrm{a}$ & $5,33 \mathrm{~b}$ & $5,98 \mathrm{a}$ & $Y=8,577-0,351 * x+0,011 * x^{2}$ & 0,91 & 15,95 \\
\hline \multicolumn{9}{|c|}{120 DIAS APÓS O ARMAZENAMENTO } \\
\hline \multicolumn{9}{|l|}{ AP07d } \\
\hline Embebição & 7,98 a & $7,75 \mathrm{a}$ & $7,55 \mathrm{a}$ & 7,46 a & $7,33 \mathrm{a}$ & $Y=7,932-0,031 * x$ & 0,97 & - \\
\hline Aplicação direta & $7,94 \mathrm{a}$ & $7,53 \mathrm{a}$ & $7,43 \mathrm{a}$ & $7,45 \mathrm{a}$ & $6,02 \mathrm{~b}$ & $Y=8,058-0,078^{*} x$ & 0,72 & - \\
\hline
\end{tabular}

Médias seguidas de mesma letra na coluna, para efeito de formas de aplicação, não diferem pelo teste de Tukey a 5\% de significância; * Significativo ao nível de 5\%; Ponto de Mínima (P. Mín.)

que no tratamento via embebição ocorreu maior valor em relação ao tratamento via aplicação direta. Para o fator doses obteve-se ajuste a uma equação de natureza linear para ambas as formas de aplicação.

$\mathrm{Na}$ Tabela 4 são apresentados os resultados da área foliar estimada no qual, não se constatou diferenças significativas quanto à forma de aplicação (aplicação direta e embebição) em todos os períodos de armazenamento avaliados. Nagashima et al. (2010), observaram que plantas provenientes de sementes tratadas tanto via embebição, quanto via aplicação direta nas doses de 3,75 e 7,5 g i.a. $\mathrm{kg}^{-1}$, aos 14 DAE apresentavam área foliar reduzida, e que esta redução foi menos acentuada com o decorrer do tempo. Esses autores relatam que aos $35 \mathrm{DAE}$ os tratamentos apresentaram diferença de área foliar em relação à testemunha e, aos $90 \mathrm{DAE}$, não foram detectadas diferenças estatísticas, demonstrando que o efeito do produto já era nulo.

Com relação ao efeito de doses, ao zero dia de armazenamento as mesmas não alteraram a área foliar estimada avaliada aos 7 DAE das plantas. Aos 14 DAE o ajuste foi de natureza linear decrescente em função do incremento das doses aplicadas. Aos 21 DAE e aos 28 DAA foi verificado ajuste quadrático em função do aumento das doses, com ponto de mínima de 14,98 e 15,10 g i.a kg-1, respectivamente. Para $120 \mathrm{DAA}$, aos 7 DAE das plantas obteve-se ajuste de natureza linear decrescente para a área foliar em função de doses. Aos 14 DAE o ajuste foi quadrático com ponto de máxima de 15,18. Já aos 21 DAE o ajuste obtido com o incremento das doses foi de natureza linear e aos 28 DAE, a área foliar ajustou-se a uma equação quadrática com ponto de máxima de 15,14.

Não houve ajuste em função de doses aos 180 DAA na avaliação realizada aos 14 DAE. Já aos 21 e 28 DAE o ajuste obtido com o incremento das doses foi de natureza linear decrescente. Os efeitos do Cloreto de Mepiquat na redução da área foliar estimada, também foram observados por Nagashima et al. (2005), utilizando sementes embebidas nas concentrações de 0,$0 ; 0,5 ; 2,5$; 5,0 e 7,5 (v/v) do produto comercial com 50 g i.a. $\mathrm{L}^{-1}$, com três períodos de imersão (3; 6 e 12 horas), no qual verificaram que, independente da dose utilizada, ocorreu redução da área foliar, em relação ao tratamento controle. Segundo Fernández et al. (1991), o Cloreto de Mepiquat inibe a expansão de folhas e pecíolos, promovendo a redução da altura da planta.

Na Tabela 5 estão apresentados os resultados de interação de formas de aplicação e doses de Cloreto de Mepiquat para as médias de área foliar estimadas aos 7; 14; 21 e 28 DAE após 60 DAA e 7 DAE após 180 DAA. As diferenças foram significativas entre as formas de aplicação na dose de $15 \mathrm{~g}$ i.a. $\mathrm{kg}^{-1}$ aos $60 \mathrm{DAA}$, com maior redução da área foliar estimada quando foi utilizada aplicação direta, em todas as avaliações realizadas.

Com relação à área foliar estimada em resposta a dose aplicada aos 7 DAE das plantas, não houve efeito significativo para o tratamento via embebição e, para o tratamento via aplicação direta, os valores se ajustaram ao modelo quadrático com ponto de mínima de 13,00 g i.a. $\mathrm{kg}^{-1}$. A área foliar estimada aos 14 DAE, tanto no tratamento via embebição quanto no via aplicação direta, decresceu linearmente em resposta às doses utilizadas. Aos 21 DAE os resultados obtidos para área foliar estimada se ajustaram tanto para o tratamento via embebição, quanto para o via aplicação direta a uma equação quadrática, com ponto de mínima de 16,09 e 13, 68 g i.a. $\mathrm{kg}^{-1}$, respectivamente. Aos 28 DAE, o ajuste foi quadrático para o tratamento via embebição, com ponto de mínima de $15,68 \mathrm{~g}$ i.a. $\mathrm{kg}^{-1} \mathrm{e}$, não significativo para o tratamento via aplicação direta. 
Tabela 4 - Área foliar estimada $\left(\mathrm{AFE} / \mathrm{cm}^{2}\right)$ avaliada aos $07 ; 14 ; 21$ e 28 DAE em plantas de algodoeiro, cultivar IPR 120 , oriundas de sementes tratadas com Cloreto de Mepiquat em função de doses e formas de aplicação em diferentes períodos de armazenamento

\begin{tabular}{|c|c|c|c|c|c|c|c|c|c|c|c|}
\hline \multicolumn{12}{|c|}{ ZERO DIA APÓS O ARMAZENAMENTO } \\
\hline \multirow{2}{*}{ Variáveis } & \multicolumn{2}{|c|}{ FORMAS } & \multicolumn{5}{|c|}{ DOSES (g i.a. $\left.\mathrm{kg}^{-1}\right)$} & \multirow[b]{2}{*}{ Equações de Regressão } & \multirow{2}{*}{$\mathrm{R}^{2}$} & \multirow{2}{*}{$\begin{array}{c}\text { P. } \\
\text { Máx. }\end{array}$} & \multirow{2}{*}{$\begin{array}{l}\text { P. } \\
\text { Mín. }\end{array}$} \\
\hline & EMB. & A.D. & 0 & 5 & 10 & 15 & 20 & & & & \\
\hline AFE07d & $15,47 \mathrm{a}$ & $14,40 \mathrm{a}$ & 15,91 & 14,95 & 14,82 & 14,22 & 13,78 & ns & - & - & - \\
\hline AFE14d & $17,01 \mathrm{a}$ & $15,35 \mathrm{a}$ & 21,21 & 17,57 & 16,47 & 15,06 & 12,11 & $Y=20,223-0,454 * x$ & 0,93 & - & - \\
\hline AFE21d & $31,78 \mathrm{a}$ & $24,03 \mathrm{a}$ & 50,85 & 30,32 & 19,83 & 19,93 & 19,21 & $Y=49,994-4,407 * x+0,147 * x^{2}$ & 0,98 & - & 14,98 \\
\hline AFE28d & $59,19 \mathrm{a}$ & $44,94 \mathrm{a}$ & 114,08 & 51,00 & 33,88 & 33,98 & 27,53 & $Y=108,77-11,265^{*} x+0,373^{*} x^{2}$ & 0,95 & - & 15,10 \\
\hline \multicolumn{12}{|c|}{120 DIAS APÓS O ARMAZENAMENTO } \\
\hline AFE07d & $11,52 \mathrm{a}$ & $11,26 \mathrm{a}$ & 13,95 & 11,68 & 10,28 & 11 & 10,08 & $\mathrm{Y}=13,079-0,168^{*} \mathrm{x}$ & 0,73 & - & - \\
\hline AFE14d & $14,48 \mathrm{a}$ & 14,03 a & 17,87 & 14,56 & 12,51 & 13,7 & 12,63 & $Y=17,629-0,668 * x+0,022 * x^{2}$ & 0,89 & 15,18 & - \\
\hline AFE21d & $17,30 \mathrm{a}$ & 15,96 a & 23,26 & 17,25 & 15,26 & 15,45 & 13,95 & $Y=21,518-0,488 * x$ & 0,86 & - & - \\
\hline AFE28d & $20,69 \mathrm{a}$ & $20,56 \mathrm{a}$ & 30,24 & 20,38 & 17,70 & 18,14 & 16,68 & $Y=29,349-1,726^{*} x-0,057^{*} x^{2}$ & 0,93 & 15,14 & - \\
\hline \multicolumn{12}{|c|}{180 DIAS APÓS O ARMAZENAMENTO } \\
\hline AFE14d & $10,96 \mathrm{a}$ & $11,30 \mathrm{a}$ & 12,71 & 11,97 & 9,84 & 10,97 & 10,19 & $\mathrm{~ns}$ & - & - & - \\
\hline AFE21d & $12,90 \mathrm{a}$ & $13,08 \mathrm{a}$ & 15,88 & 14,44 & 10,48 & 12,61 & 11,58 & $\mathrm{Y}=15,085-0,209 * \mathrm{x}$ & 0,58 & - & - \\
\hline AFE28d & $18,84 \mathrm{a}$ & $21,14 \mathrm{a}$ & 27,24 & 23,25 & 15,62 & 18,04 & 15,80 & $Y=25,609-0,562 * x$ & 0,76 & - & - \\
\hline
\end{tabular}

Médias seguidas de mesma letra na linha, para efeito de formas de aplicação, não diferem pelo teste de Tukey a 5\% de significância; * Significativo a 5\%; ns: não significativo; Embebição (EMB.); Aplicação Direta (A.D.); Ponto de Máxima (P. Máx.); Ponto de Mínima (P. Mín.)

Tabela 5 - Interação de formas (FA) e doses para médias da área foliar estimada (AFE/ $\mathrm{cm}^{2}$ ), avaliadas aos 07, 14, 21 e $28 \mathrm{DAE}$, em plantas de algodoeiro, cultivar IPR 120, oriundas de sementes tratadas com Cloreto de Mepiquat, aos 60 e 180 dias após armazenamento

\begin{tabular}{|c|c|c|c|c|c|c|c|c|}
\hline \multicolumn{9}{|c|}{60 DIAS APÓS O ARMAZENAMENTO } \\
\hline \multicolumn{7}{|c|}{ DOSES (g i.a. $\mathrm{kg}^{1}{ }^{1}$ ) } & \multirow{2}{*}{$\mathrm{R}^{2}$} & \multirow{2}{*}{$\begin{array}{c}\text { P. } \\
\text { Mín. }\end{array}$} \\
\hline FA & 0 & 5 & 10 & 15 & 20 & Equação de Regressão & & \\
\hline Embebição & $24,47 \mathrm{a}$ & $20,19 \mathrm{a}$ & $16,36 \mathrm{a}$ & $23,80 \mathrm{a}$ & $20,20 \mathrm{a}$ & ns & - & - \\
\hline \multicolumn{9}{|l|}{ AF14d } \\
\hline Embebição & $90,05 \mathrm{a}$ & 54,24 a & 39,44 a & $67,70 \mathrm{a}$ & 36,42 a & $Y=76,328-1,876 * x$ & 0,45 & - \\
\hline $\begin{array}{c}\text { Aplicação direta } \\
\text { AF21d }\end{array}$ & $72,93 \mathrm{a}$ & $55,36 \mathrm{a}$ & 48,56 a & $42,34 \mathrm{~b}$ & $38,70 \mathrm{a}$ & $Y=67,869-2,229 * x$ & AF21d & - \\
\hline Embebição & 206,57 a & 90,44 a & 67,91 a & $133,89 \mathrm{a}$ & $57,62 \mathrm{a}$ & $\mathrm{Y}=189,805-14,181 * \mathrm{x}+0,440 * \mathrm{x}^{2}$ & 0,62 & 16,09 \\
\hline \multicolumn{9}{|l|}{ AF28d } \\
\hline Embebição & $411,28 \mathrm{a}$ & $207,84 \mathrm{a}$ & $114,55 \mathrm{a}$ & 277,28 a & $110,04 \mathrm{a}$ & $\mathrm{Y}=377,721-29,938 * \mathrm{x}+0,938 * \mathrm{x}^{2}$ & 0,57 & 15,68 \\
\hline Aplicação direta & $391,40 \mathrm{a}$ & $254,45 \mathrm{a}$ & 153,39 a & $131,65 \mathrm{~b}$ & $183,14 \mathrm{a}$ & $\mathrm{ns}$ & - & - \\
\hline \multicolumn{9}{|c|}{180 DIAS APÓS O ARMAZENAMENTO } \\
\hline \multicolumn{9}{|l|}{ AF7d } \\
\hline Embebição & $8,86 \mathrm{a}$ & $8,90 \mathrm{a}$ & $7,16 \mathrm{a}$ & 7,89 a & $7,44 \mathrm{a}$ & ns & - & - \\
\hline Aplicação direta & $9,59 \mathrm{a}$ & $7,68 \mathrm{a}$ & $7,10 \mathrm{a}$ & $9,53 \mathrm{a}$ & $4,05 \mathrm{~b}$ & $Y=9,432-0,184 * x$ & 0,44 & - \\
\hline
\end{tabular}

Médias seguidas de mesma letra na coluna, para efeito de formas de aplicação, não diferem pelo teste de Tukey a 5\% de significância; * Significativo a 5\%; ns: não significativo ao nível de 5\%; Ponto de Mínima (P. Mín.) 
O resultado obtido em função de doses no tratamento via embebição para a variável área foliar estimada aos 7 DAE, não foram significativos aos 180 DAA das sementes tratadas. Para o tratamento via aplicação direta obteve-se ajuste linear decrescente em função do aumento das doses. Tais dados estão de acordo com os verificados por Nagashima et al. (2005), os quais observaram que independente da dose utilizada do regulador, ocorreu redução da área foliar por planta e por folha de algodoeiro, cultivar IPR 120.

Na Tabela 6 constam os resultados das avaliações do diâmetro do caule, da matéria seca de caule e de folhas aos 28 DAE para os períodos de zero; 60; 120 e 180 dias de armazenamento. Foram verificados efeitos significativos quanto à forma de aplicação do Cloreto de Mepiquat apenas para o diâmetro do caule aos 120 e 180 DAA, com valores inferiores para o tratamento via aplicação direta em relação à embebição.

Ao zero e 60 dias de armazenamento não houve efeito de doses para o diâmetro do caule. Contudo, aos 120 e 180 DAA, os ajustes para o diâmetro foram quadráticos em função do aumento das doses, com ponto de máxima de 10,12 e ponto de mínima 4,0 g i.a. $\mathrm{kg}^{-1}$, respectivamente.

O uso do regulador de crescimento proporcionou menor matéria seca de caules em todas as doses utilizadas, independentes do período de armazenamento, quando comparadas à testemunha (TAB. 6). Na avaliação de 0 DAA, a matéria seca do caule ajustou-se ao modelo quadrático em função do aumento das doses, com ponto de mínima de 14,10 g i.a kg-1. Aos 60 DAA, os valores se ajustaram a uma equação linear decrescente e aos 120 e 180 DAA não houve efeito de dose.

Com relação à matéria seca de folhas ao zero e 120 DAA das sementes tratadas (TAB. 6), observou-se efeito de doses com ajuste linear decrescente em resposta ao aumento das mesmas e, aos 180 DAA não se verificou diferença significativa. Os resultados confirmam os obtidos por Nagashima et al. (2005), que avaliaram o efeito do tratamento de sementes de algodão, via embebição com o Cloreto de Mepiquat, visando o controle do crescimento da planta desde a emergência e, também os resultados observados por Zhao e Oosterhuis (2000) ao utilizarem o mesmo regulador de crescimento via aplicação foliar.

Aos 120 e 180 dias após tratamento de sementes, quando comparados aos demais períodos de armazenamento, foram obtidas plantas com valores inferiores em todas as características avaliadas, inclusive para o tratamento testemunha na qual não houve aplicação de regulador de crescimento (TAB. 6). Estes resultados

Tabela 6 - Diâmetro do caule (DC), matéria seca de caules (MSC) e de folhas (MSF), avaliados aos 28 DAE em plantas de algodoeiro, cultivar IPR 120, oriundas de sementes tratadas com Cloreto de Mepiquat em função de formas e doses em diferentes períodos de armazenamento

\begin{tabular}{|c|c|c|c|c|c|c|c|c|c|c|c|}
\hline \multicolumn{12}{|c|}{ ZERO DIA APÓS O ARMAZENAMENTO } \\
\hline \multirow[t]{2}{*}{ Variáveis } & \multicolumn{2}{|c|}{ FORMAS } & \multirow[b]{2}{*}{0} & \multicolumn{5}{|c|}{ DOSES (g i.a. $\mathrm{kg}^{-1}$ ) } & \multirow[b]{2}{*}{$\mathrm{R}^{2}$} & \multirow[b]{2}{*}{$\begin{array}{c}\text { P. } \\
\text { Máx. }\end{array}$} & \multirow[b]{2}{*}{$\begin{array}{l}\text { P. } \\
\text { Mín. }\end{array}$} \\
\hline & EMB. & A.D. & & 5 & 10 & 15 & 20 & Equações de Regressão & & & \\
\hline $\mathrm{DC}(\mathrm{mm})$ & $2,65 \mathrm{a}$ & $2,45 \mathrm{a}$ & 2,81 & 2,59 & 2,54 & 2,24 & 2,58 & $\mathrm{~ns}$ & - & - & - \\
\hline $\operatorname{MSC}(g)$ & $0,70 \mathrm{a}$ & $0,55 \mathrm{a}$ & 0,138 & 0,066 & 0,043 & 0,038 & 0,041 & $Y=0,1334-0,0141 * x+0,0005 * x^{2}$ & 0,98 & - & 14,10 \\
\hline $\operatorname{MSF}(\mathrm{g})$ & $59,19 \mathrm{a}$ & $44,94 \mathrm{a}$ & 114,08 & 51,00 & 43,88 & 33,88 & 27,53 & $Y=0,2488+0,0066^{*} x$ & 0,82 & - & - \\
\hline \multicolumn{12}{|c|}{60 DIA APÓS O ARMAZENAMENTO } \\
\hline $\mathrm{DC}(\mathrm{mm})$ & $3,72 \mathrm{a}$ & $3,57 \mathrm{a}$ & 3,76 & 3,74 & 3,43 & 3,85 & 3,45 & ns & - & - & - \\
\hline $\operatorname{MSC}(g)$ & $0,580 \mathrm{a}$ & $0,510 \mathrm{a}$ & 0,808 & 0,579 & 0,393 & 0,624 & 0,344 & $Y=0,726-0,018^{*} x$ & 0,56 & - & - \\
\hline \multicolumn{12}{|c|}{120 DIA APÓS O ARMAZENAMENTO } \\
\hline $\mathrm{DC}(\mathrm{mm})$ & $2,64 \mathrm{a}$ & $2,31 \mathrm{~b}$ & 2,35 & 2,60 & 2,59 & 2,73 & 2,14 & $Y=2,322+0,081 * x-0,004 * x^{2}$ & 0,80 & 10,12 & - \\
\hline $\operatorname{MSC}(\mathrm{g})$ & $0,050 \mathrm{a}$ & $0,040 \mathrm{a}$ & 0,051 & 0,055 & 0,043 & 0,04 & 0,048 & ns & - & - & - \\
\hline $\operatorname{MSF}(\mathrm{g})$ & $0,110 \mathrm{a}$ & $0,120 \mathrm{a}$ & 0,154 & 0,118 & 0,113 & 0,11 & 0,106 & $\mathrm{Y}=0,140-0,002 * \mathrm{x}$ & - & - & - \\
\hline \multicolumn{12}{|c|}{180 DIA APÓS O ARMAZENAMENTO } \\
\hline $\mathrm{DC}(\mathrm{mm})$ & $2,09 \mathrm{a}$ & $2,06 \mathrm{~b}$ & 2,08 & 2,05 & 2,03 & 2,1 & 2,13 & $Y=2,073-0,008 * x+0,001 * x^{2}$ & 0,84 & - & 4,00 \\
\hline $\operatorname{MSC}(\mathrm{g})$ & $0,030 \mathrm{a}$ & $0,020 \mathrm{a}$ & 0,03 & 0,034 & 0,021 & 0,023 & 0,026 & ns & - & - & - \\
\hline $\operatorname{MSF}(\mathrm{g})$ & $0,090 \mathrm{a}$ & $0,080 \mathrm{a}$ & 0,133 & 0,098 & 0,065 & 0,061 & 0,084 & ns & - & - & - \\
\hline
\end{tabular}

Médias seguidas de mesma letra na linha, para efeito de formas de aplicação, não diferem pelo teste de Tukey a 5\% de significância; *Significativo a 5\%; ns: não significativo ao nível de 5\%; Embebição (EMB.); Aplicação Direta (A.D.) 
estão relacionados ao período de baixas temperaturas em que foi conduzida a avaliação, uma vez que o experimento foi iniciado em dezembro, período do ano com temperaturas médias elevadas, e, com o decorrer do armazenamento as últimas avaliações foram conduzidas em julho, período de temperaturas mais amenas para a região.

Segundo Beltrão (2006) o algodoeiro, necessita de temperaturas entre 27 e $32{ }^{\circ} \mathrm{C}$, para se ter um bom desenvolvimento e, a temperatura na casa de vegetação neste período variou entre 11,5 e $16{ }^{\circ} \mathrm{C}$. No entanto, mesmo no período de temperaturas mais amenas a diferença entre doses permaneceu evidente, seguindo comportamento semelhante ao observado nos primeiros períodos de armazenamento, quando relacionadas com a testemunha. Reddy et al. (1990), verificaram que o maior efeito do produto no crescimento do algodoeiro ocorreu quando a temperatura diurna foi de $30^{\circ} \mathrm{C}$, com noites de $20^{\circ} \mathrm{C}$ e, em temperaturas maiores ou menores que estas o efeito do produto era nulo ou se tornava menos pronunciado.

Quanto aos resultados de interação de formas de aplicação e doses de Cloreto de Mepiquat para a matéria seca de folhas (MSF/g) aos 60 DAA das sementes tratadas foram verificadas diferenças significativas quanto à forma de aplicação com valores inferiores para o tratamento via aplicação direta em relação à embebição quando utilizado a dose de 15 g i.a. $\mathrm{kg}^{-1}$ (TAB. 7). Para o tratamento via embebição os resultados se ajustaram a uma equação linear decrescente com o incremento das doses e, para o tratamento via aspersão não houve efeito de doses aplicadas.

Os resultados obtidos corroboram com Lamas et al. (2000) que confirmam a redução de matéria seca de folhas, por meio de pulverizações foliares parceladas e, com Nagashima et al. (2005), que ao utilizar sementes tratadas via embebição, observaram que o uso de Cloreto de Mepiquat em doses acima de 2,5\%, reduz a área foliar e a matéria seca da parte aérea, observadas aos 49 DAE e, também, o número de ramos e estruturas reprodutivas.

As formas de aplicação utilizadas (aplicação direta e embebição) demonstraram resultados semelhantes e positivos com relação à redução do porte da planta, da área foliar e da matéria seca de caules e folhas. O uso de redutores de crescimento na cultura do algodoeiro é crescente em virtude dos sistemas de produção utilizado na cultura atualmente e, a obtenção de plantas com menor porte é fundamental, principalmente, para o sucesso do sistema adensado de cultivo.

Tradicionalmente, a aplicação do regulador de crescimento é realizada via aplicação foliar, contudo, esse método apresenta vários inconvenientes como a perda do produto, caso ocorra chuvas após a aplicação e, a necessidade de várias aplicações para um controle efetivo da altura das plantas, onerando assim os custos de produção. Dessa forma, os resultados obtidos confirmam a possibilidade de uso do tratamento de sementes como forma de aplicação do Cloreto de Mepiquat, assegurando um controle do crescimento desde o início do desenvolvimento da planta. Dentre as formas de aplicação via sementes, embora não tenha ocorrido diferença nos resultados proporcionados pelas mesmas, a aplicação direta apresenta a vantagem de ser mais rápida a execução e, devido ao menor volume de solução utilizado, facilita o processo de secagem após o tratamento. Embora ainda careça de estudos, o método da aplicação direta oferece, ainda, a possibilidade de poder ser conciliado com o tratamento de sementes com fungicida, normalmente realizado previamente à semeadura ou ao armazenamento das sementes de algodão. Como constatado, o tratamento com o regulador pode ser realizado antes do armazenamento das sementes, independente da dose, sem que haja perda da sua ação reguladora, facilitando a comercialização e a operação de semeadura.

Tabela 7 - Interação de formas (FA) e doses para médias de matéria seca de folhas (MSF) aos 60 DAA de plantas de algodoeiro, cultivar IPR 120, oriundas de sementes tratadas com Cloreto de Mepiquat

\begin{tabular}{cccccccc}
\hline \multicolumn{7}{c}{ 60 DIAS APÓS O ARMAZENAMENTO } \\
\hline MSF/g & 0 & 5 & 10 & 15 & 20 & Equação de Regressão & $\mathrm{R}^{2}$ \\
\hline FA & $1,540 \mathrm{a}$ & $0,973 \mathrm{a}$ & $0,808 \mathrm{a}$ & $0,598 \mathrm{~b}$ & $0,540 \mathrm{a}$ & $\mathrm{Y}=1,286-0,032^{*} \mathrm{x}$ & 0,35 \\
Embebição & $0,975 \mathrm{a}$ & $0,710 \mathrm{a}$ & $0,720 \mathrm{a}$ & $0,750 \mathrm{a}$ & $\mathrm{ns}$ & - \\
\hline
\end{tabular}

Médias seguidas de mesma letra na coluna, para efeito de formas de aplicação, não diferem pelo teste de Tukey a 5\% de significância; *Significativo a $5 \%$; ns: não significativo a $5 \%$ 


\section{Conclusões}

1. Independente da forma de aplicação via semente, o Cloreto de Mepiquat reduz o diâmetro do caule, a matéria seca de folhas e caule, a área foliar, e a altura de plantas de algodoeiro até os 28 dias após a emergência, com efeitos intensificados com o aumento das doses aplicadas;

2. A forma de aplicação via sementes e o armazenamento das sementes tratadas por até 180 dias não interferem na ação do regulador de crescimento em plantas de algodão até os 28 dias após a emergência;

3. A ação reguladora de crescimento do Cloreto de Mepiquat perdura, pelo menos, até aos 28 dias após a emergência das plantas, independente da forma de aplicação, dose utilizada e período de armazenamento das sementes tratadas.

\section{Referências}

BELTRÃO, N. E. M. Clima regula produção e qualidade da fibra do algodoeiro. Visão Agrícola, n. 06,p. 76-77, 2006.

BELTRÃO, N. E. de M. et al. Recomendações técnicas e condições gerais sobre o uso de herbicidas, desfolhantes e reguladores de crescimento na cultura do algodão. Campina Grande: EMBRAPA - CPNA, 1997. 32 p. (EMBRAPA-CPNA. Documentos, 48).

FERNANDEZ, C. J.; COTHREN, J. T.; McINNES, K. J. Partitioning of biomass in well-watered and water-stressed cotton plants treated with mepiquat chloride. Crop Science, v. 31, n. 05, p. 1224-1228, 1991 .

HOLDEN, J. et al. The use of Pix as a cotton management tool. Australian Cotton CRC. 2004. Disponível em: <http:// www.cotton. Crc.org/au/publicat/agro/pix.htm>. Acesso em: 25 abr. 2009.

LAMAS, F. M.; ATHAYDE, M. L.; BANZATTO, D. A. Reações do algodoeiro CNPA-ITA 90 ao cloreto de mepiquat. Pesquisa Agropecuária Brasileira, v. 35, n. 05, p. 507-516, 2000.

LAMAS, F. M. Cloreto de mepiquat na cultura do algodoeiro via sementes. Dourados: Embrapa Agropecuária Oeste, 2006. 19 p. (Boletim de Pesquisa e Desenvolvimento, 33). Disponível em: $\quad<$ http://www.cpao.embrapa.br/publicacoes/ficha. php?tipo=BP\&num=33 \&ano=2006 $>$. Acesso em: 30 abr. 2009.

MARCOS FILHO, J. Teste de envelhecimento acelerado. In: KRZYZANOWSKI, F. C.; VIEIRA, R. D.; FRANÇA NETO, J. B. (ed.). Vigor de sementes: conceitos e testes. Londrina: ABRATES, 1999. cap. 3, p. 1-24.

MATEUS, G. P.; LIMA, E. V.; ROSOLEM, C. A. Perdas de cloreto de mepiquat no algodoeiro por chuva simulada. Pesquisa Agropecuária Brasileira, v. 39, n. 07, p. 631-636, 2004.

NAGASHIMA, G. T. et al. Desenvolvimento do algodoeiro em resposta a modo de aplicação e doses de cloreto de mepiquat via sementes. Ciência Rural, v. 40, n. 01 p. 7-11, 2010.

NAGASHIMA, G. T. et al. Cloreto de mepiquat via embebição de sementes e aplicação foliar em algodoeiro em espaçamento ultraestreito. Revista Ciência Agronômica, v. 40, n. 04, p. 602609, 2009.

NAGASHIMA, G. T. et al. Embebição de sementes e aplicação foliar com cloreto de mepiquat no crescimento e produção do algodoeiro. Ciência e Agrotecnologia, v. 31, n. 04, p. 10271034, 2007.

NAGASHIMA, G. T. et al. Desenvolvimento de plantas de algodão provenientes de sementes embebidas em cloreto de mepiquat. Pesquisa Agropecuária Brasileira, v. 40, n. 09, p. 943-946, 2005.

REDDY, V. R; BAKER, D. N.; HODGES, H. F. Temperature and mepiquat chloride on cotton canopy architecture. Agronomy Journal, v. 82, n. 02, p. 190-195, 1990.

YEATS, S. J.; CONSTABLE, G. A.; McCUMSTIE, T. Cottton growth and yield after seed treatment with mepiquat chloride in tropical winter season. Field Crops Research, v. 93, n. 02/03, p. 122-131, 2005 .

ZHAO, D.; OOSTERHUIS, D. M.; DANIEL, T. Two-year study on the efficacy of PIX $^{\mathrm{TM}}$ when foliar application is followed by precipitation. In: BELTWIDE COTTON PRODUCTION RESEARCH CONFERENCES, 2000, Proceedings... Memphis: National Cotton Council of America, Memphis, 2000. p. 681-684. v. 1.

ZANQUETA, R. et al. Modos de aplicação de regulador de crescimento com diferentes densidades de plantas em cultivares de algodão herbáceo (Gossypium hirtisutum L. Latifolium Hutch.) Acta Scientiarum Agronomy, v. 26, n. 01, p. 97-105, 2004. 